\title{
A Note on Closed-Form Representation of Fibonacci Numbers Using Fibonacci Trees
}

\author{
Indhumathi Raman \\ School of Information Technology and Engineering, VIT University, Vellore 632014, India \\ Correspondence should be addressed to Indhumathi Raman; indhumathi.raman@gmail.com
}

Received 7 November 2013; Accepted 22 December 2013; Published 12 February 2014

Academic Editors: M. Kano and X. Zhang

Copyright ( 2014 Indhumathi Raman. This is an open access article distributed under the Creative Commons Attribution License, which permits unrestricted use, distribution, and reproduction in any medium, provided the original work is properly cited.

We give a new representation of the Fibonacci numbers. This is achieved using Fibonacci trees. With the help of this representation, the $n$th Fibonacci number can be calculated without having any knowledge about the previous Fibonacci numbers.

\section{Introduction}

A Fibonacci tree is a rooted binary tree in which for every nonleaf vertex $v$, the heights of the subtrees, rooted at the left and right child of $v$, differ by exactly one. A formal recursive definition of the Fibonacci tree (denoted by $\mathbb{F}_{h}$ if its height is $h)$ is given below.

Definition 1. $\mathbb{F}_{0}:=K_{1}, \mathbb{F}_{1}:=K_{2}$. For $h \geq 2, \mathbb{F}_{h}$ is obtained by taking a copy of $\mathbb{F}_{h-1}$, a copy of $\mathbb{F}_{h-2}$, a new vertex $R$, and joining $R$ to the roots of $\mathbb{F}_{h-1}$ and $\mathbb{F}_{h-2}$.

Figure 1 shows this construction and a few small Fibonacci trees.

The above recursive definition implies that the number of vertices in $\mathbb{F}_{h}$ is $\left|V\left(\mathbb{F}_{h}\right)\right|=\left|V\left(\mathbb{F}_{h-1}\right)\right|+\left|V\left(\mathbb{F}_{h-2}\right)\right|+1$. On solving this recurrence relation, we get $\left|V\left(\mathbb{F}_{h}\right)\right|=f(h+2)$ 1 , where $f(i)$ is the $i$ th number in the Fibonacci sequence, $f(0)=1, f(1)=1, f(n)=f(n-1)+f(n-2)$; this justifies the terminology Fibonacci tree.

The Fibonacci tree is the one with the minimum number of vertices among the class of AVL trees (see [1]). Several properties of Fibonacci trees have been investigated: for example, Fibonacci numbers of Fibonacci trees have been studied in [2], optimality of Fibonacci numbers is discussed in [3], asymptotic properties of Balaban's index for Fibonacci trees have been explored in [4], and Zeckendorf representation of integers is given in [5]. In this short paper, we represent the number of vertices of $\mathbb{F}_{h}$ in closed form (A closed form is one which gives the value of a sequence at index $n$ in terms of only one parameter, $n$ itself.) by observing the number of vertices at each level of $\mathbb{F}_{h}$. Such a calculation helps us to give a closed-form representation of $n$th Fibonacci number for every $n \geq 2$.

\section{Closed-Form Representation of Fibonacci Numbers}

There are several closed-form representations of the Fibonacci numbers. We state a few below.

(i) Consider

$$
f(n)=\frac{(1+\sqrt{5})^{n}-(1-\sqrt{5})^{n}}{2^{n} \sqrt{5}} .
$$

It was also derived by Binet (see [6]) in 1843, although the result was known to Euler, Daniel Bernoulli, and de Moivre more than a century earlier.

(ii) Consider

$$
B(x)=\sum_{k=0}^{\infty} b_{k} x^{k}
$$

In the above generating function for the Fibonacci numbers the value of $b_{k}$ gives the $k$ th Fibonacci number. However, expanding the generating function involves tedious calculations. 
(iii) Consider

$$
f_{n}=\operatorname{round}\left(\frac{5+\sqrt{5}}{10}\left(\frac{1+\sqrt{5}}{2}\right)^{n}\right) \text {. }
$$

It was also derived by Binet (see [6]) where the function round() rounds the simplified expression up or down to an integer.

In this section, we give a simpler closed-form combinatorial representation of Fibonacci numbers. To do so, we first give a closed-form representation of the number of vertices $\left|V\left(\mathbb{F}_{h}\right)\right|$ of $\mathbb{F}_{h}$ (the Fibonacci tree of height $h$ ). The following lemma gives the number of vertices in a particular level of $\mathbb{F}_{h}$ and thereafter we sum the number of vertices over the levels to get $\left|V\left(\mathbb{F}_{h}\right)\right|$.

Lemma 2. Let $\mathbb{F}_{h}$ be a Fibonacci tree of height $h$ and let $k$ be an integer such that $0 \leq k \leq h$. The number of vertices $N(h, k)$ at level $k$ of $\mathbb{F}_{h}$ is given by

$$
N(h, k)=\sum_{i=0}^{h-k}\left(\begin{array}{c}
k \\
h-k-i
\end{array}\right) .
$$

Proof. We prove the lemma by induction on $k$. For $k=0$ we have $N(h, 0)=\sum_{i=0}^{h}\left(\begin{array}{c}0 \\ h-i\end{array}\right)$. Using the convention $\left(\begin{array}{c}n \\ r\end{array}\right)=$ 0 if $n<r$, we have $N(h, 0)=\left(\begin{array}{l}0 \\ 0\end{array}\right)=1$. This is true since the root of $\mathbb{F}_{h}$ is the only vertex at level 0 . Further proceeding, from the recursive definition of $\mathbb{F}_{h}$, we have

$$
\begin{aligned}
N & (h, k) \\
= & N(h-1, k-1)+N(h-2, k-1) \\
= & \sum_{i=0}^{h-k}\left(\begin{array}{c}
k-1 \\
h-k-i
\end{array}\right)+\sum_{j=0}^{h-k-1}\left(\begin{array}{c}
k-1 \\
h-k-j-1
\end{array}\right) \\
= & \sum_{i=0}^{h-k}\left(\begin{array}{c}
k-1 \\
h-k-i
\end{array}\right)+\sum_{j=0}^{h-k}\left(\begin{array}{c}
k-1 \\
h-k-j-1
\end{array}\right) \\
& -\left(\begin{array}{c}
k-1 \\
-1
\end{array}\right) \\
= & \left.\sum_{i=0}^{h-k}\left(\begin{array}{c}
k-1 \\
h-k-i
\end{array}\right)+\left(\begin{array}{c}
k-1 \\
h-k-i-1
\end{array}\right)\right) \quad \text { since }\left(\begin{array}{c}
n \\
r
\end{array}\right)=0 \\
= & \sum_{i=0}^{h-k}\left(\begin{array}{c}
k \\
h-k-i
\end{array}\right) .
\end{aligned}
$$

In Step 3 of the above equation, we add and subtract $\left(\begin{array}{c}k-1 \\ h-k-j-1\end{array}\right)$ for $j=h-k$. This proves the lemma.

The number of vertices in any tree is the sum of the vertices at its levels. In particular, $\left|V\left(\mathbb{F}_{h}\right)\right|=\sum_{k=0}^{h} N(h, k)$. Hence we have the following lemma.

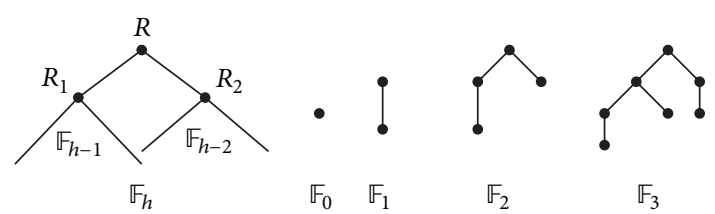

FIgURE 1: Recursive construction and examples of Fibonacci Trees.

Lemma 3. Let $\mathbb{F}_{h}$ be the Fibonacci tree of height $h$; then the number of vertices $\left|V\left(\mathbb{F}_{h}\right)\right|$ of $\mathbb{F}_{h}$ is $\sum_{k=0}^{h} \sum_{i=0}^{h-k}\left(\begin{array}{c}k \\ h-k-i\end{array}\right)$.

The above theorem helps us to derive a closed-form representation of the Fibonacci numbers. This representation is in contrast to the recurrence relation form, which has certain previous values of the sequence as parameters. We know that $\left|V\left(\mathbb{F}_{h}\right)\right|=f(h+2)-1$. Equivalently $f(n)=$ $1+\left|V\left(\mathbb{F}_{n-2}\right)\right|$.

Theorem 4. Let $f(n)$ be the nth number in the Fibonacci sequence starting with $f(0)=1$ and $f(1)=1$. Then for $n \geq 2$,

$$
f(n)=1+\sum_{k=0}^{n-2} \sum_{i=0}^{n-k-2}\left(\begin{array}{c}
k \\
n-k-i-2
\end{array}\right) .
$$

Proof. Since $f(n)=\left|V\left(\mathbb{F}_{\{n-2\}}\right)\right|+1$, the proof is an immediate consequence of Lemma 3.

As an example for Theorem 4, we calculate $f(4)$ and $f(5)$ :

$$
\begin{aligned}
f(4)= & 1+\sum_{k=0}^{2} \sum_{i=0}^{2-k}\left(\begin{array}{c}
k \\
2-k-i
\end{array}\right) \\
= & 1+\sum_{i=0}^{2}\left(\begin{array}{c}
0 \\
2-i
\end{array}\right)+\sum_{i=0}^{1}\left(\begin{array}{c}
1 \\
1-i
\end{array}\right)+\sum_{i=0}^{0}\left(\begin{array}{c}
2 \\
0-i
\end{array}\right) \\
= & 1+\left(\begin{array}{l}
0 \\
0
\end{array}\right)+\left(\begin{array}{l}
1 \\
1
\end{array}\right)+\left(\begin{array}{l}
1 \\
0
\end{array}\right)+\left(\begin{array}{l}
2 \\
0
\end{array}\right) \\
= & 5, \\
f(5)= & 1+\sum_{k=0}^{3} \sum_{i=0}^{3-k}\left(\begin{array}{c}
k-k-i \\
3-k
\end{array}\right) \\
= & 1+\sum_{i=0}^{3}\left(\begin{array}{c}
0 \\
3-i
\end{array}\right)+\sum_{i=0}^{2}\left(\begin{array}{c}
1 \\
2-i
\end{array}\right) \\
& +\sum_{i=0}^{1}\left(\begin{array}{c}
2 \\
1-i
\end{array}\right)+\sum_{i=0}^{0}\left(\begin{array}{c}
3 \\
0-i
\end{array}\right) \\
= & 1+\left(\begin{array}{l}
0 \\
0
\end{array}\right)+\left(\begin{array}{l}
1 \\
1
\end{array}\right)+\left(\begin{array}{l}
1 \\
0
\end{array}\right)+\left(\begin{array}{l}
2 \\
1
\end{array}\right)+\left(\begin{array}{l}
2 \\
0
\end{array}\right)+\left(\begin{array}{l}
3 \\
0
\end{array}\right) \\
= & 8 .
\end{aligned}
$$

\section{Conclusion}

In this paper, we give a closed-form representation of Fibonacci numbers using Fibonacci trees. A similar approach 
can be attempted for finding a closed-form representation for Lucas and Bernoulli numbers.

\section{Conflict of Interests}

The author declares that there is no conflict of interests regarding the publication of this paper.

\section{References}

[1] G. M. Adelson-Velskii and E. M. Landis, "An algorithm for organization of information," Soviet Mathematics. Doklady, vol. 3, pp. 1259-1262, 1962.

[2] S. G. Wagner, "The Fibonacci number of Fibonacci trees and a related family of polynomial recurrence systems," Fibonacci Quarterly, vol. 45, no. 3, pp. 247-253, 2007.

[3] Y. Horibe, "Notes on Fibonacci trees and their optimality," The Fibonacci Quaterly, vol. 21, no. 2, pp. 118-128, 1983.

[4] N. Jia and K. W. McLaughlin, "Fibonacci trees: A study of the asymptotic behavior of Balaban's index," MATCH. Communications in Mathematical and in Computer Chemistry, no. 51, pp. 79-95, 2004.

[5] R. M. Capocelli, "A note on Fibonacci trees and the Zeckendorf representation of integers," The Fibonacci Quarterly, vol. 26, no. 4, pp. 318-324, 1988.

[6] W. Eric, "Binet's Fibonacci Number Formula," from MathWorld. 


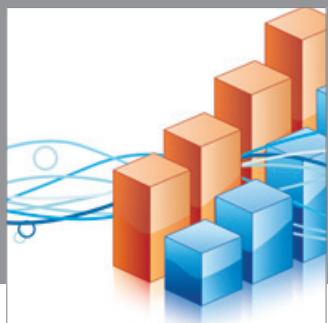

Advances in

Operations Research

mansans

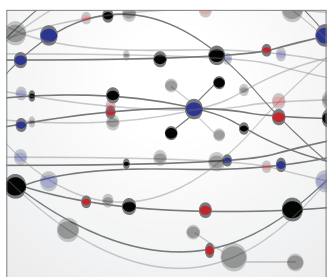

The Scientific World Journal
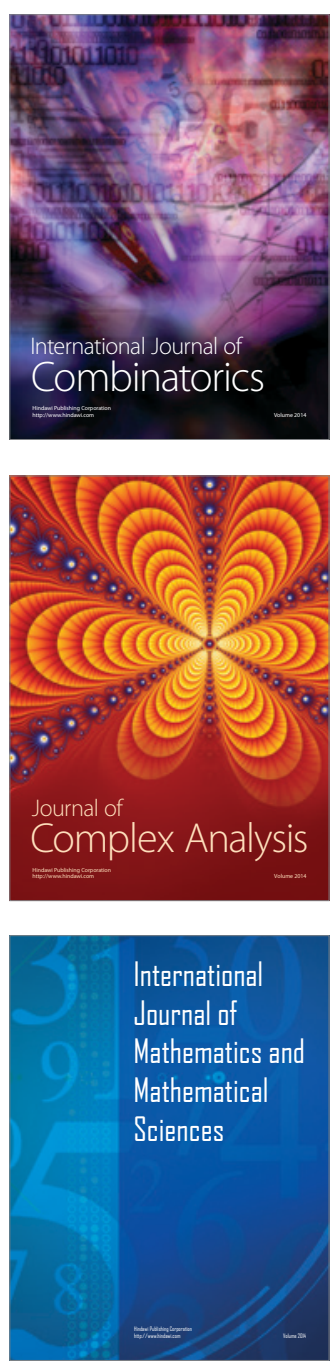
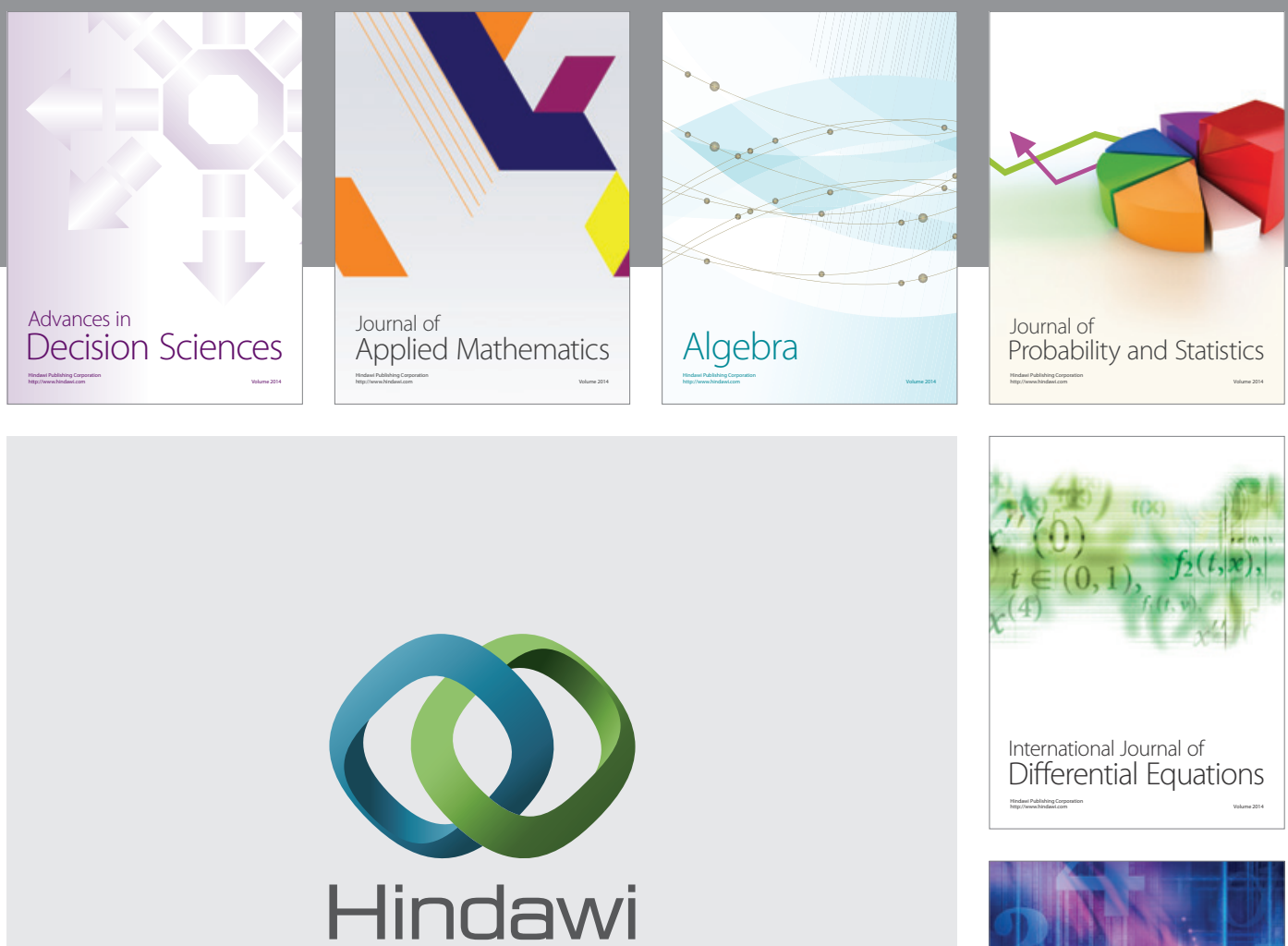

Submit your manuscripts at http://www.hindawi.com
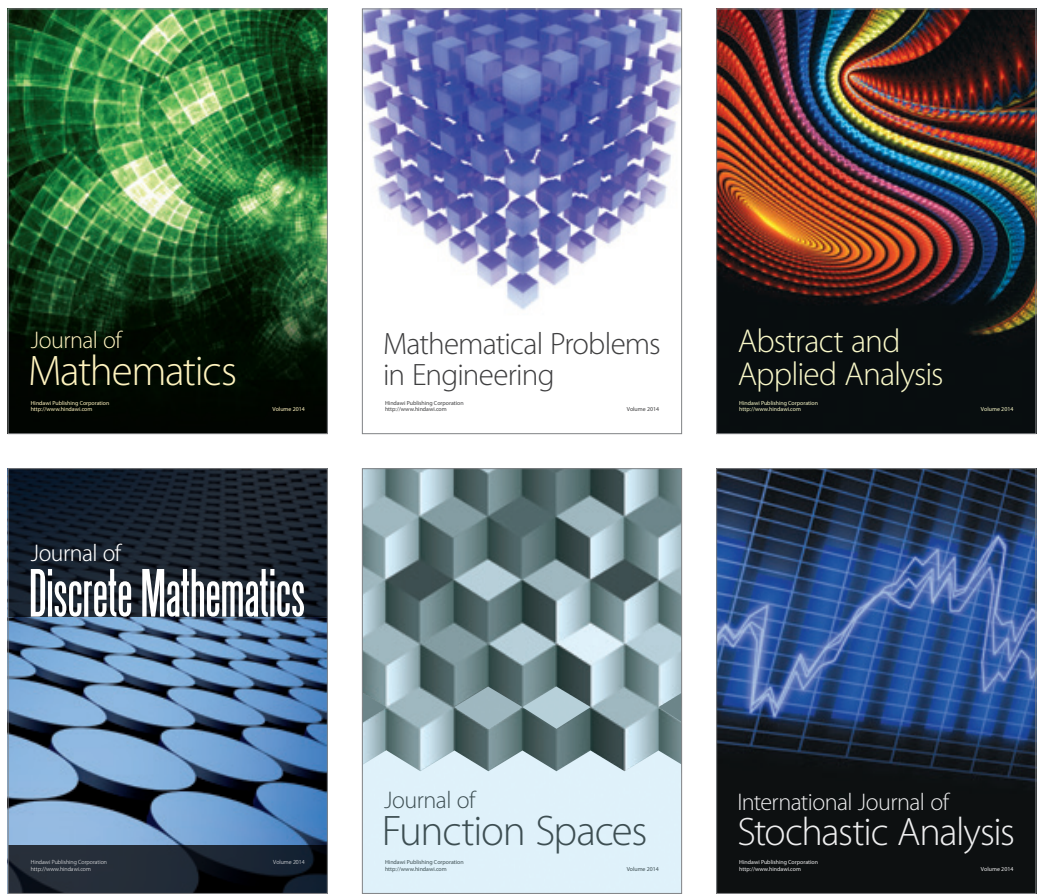

Journal of

Function Spaces

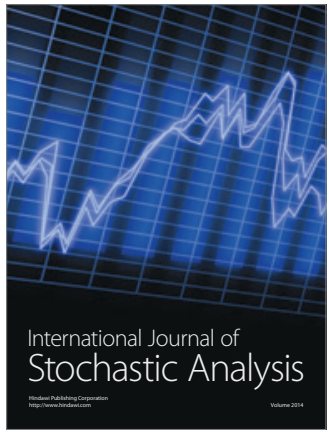

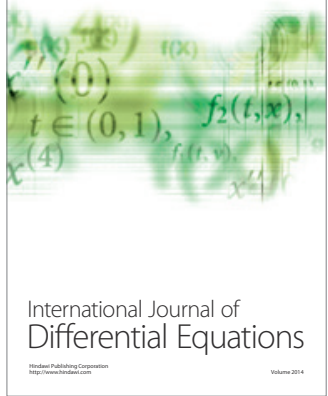
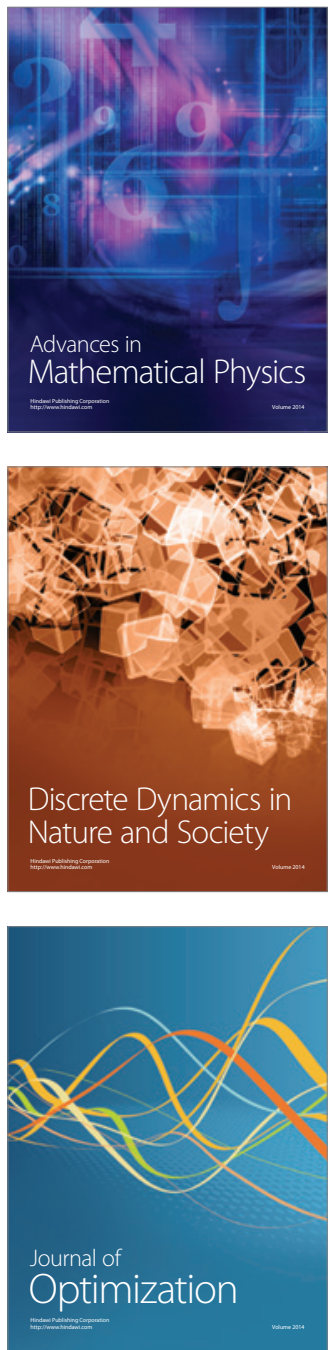